\title{
Microthermoelectric devices keep their cool
}

Thermoelectric technology converts heat into electricity or electricity into cooling. It typically uses devices composed of thermoelectric 'legs' sandwiched between two thermally conducting substrates; the legs are made from n-type and p-type semiconductors, pairs of which are electrically connected in series using alternating top and bottom metal contacts. In thermoelectric coolers, an electric current is passed through the device, forcing heat to flow from one side to the other, creating a temperature difference between the two substrates.

Microthermoelectric coolers, which have legs with smaller cross-sections and heights than conventional devices, can be used to locally manage heat in electronic circuits and have been under development for around 20 years. To be practical, the devices should be compatible with the microelectromechanical systems (MEMS) found in modern electronics, which are fabricated using electrochemical deposition and photolithography. However, creating efficient microthermoelectric coolers using fabrication methods that are both MEMScompatible and economical has proved challenging. Guodong Li and colleagues at IFW Dresden, Dresden University of Technology, and Consejo Nacional de Ciencia y Tecnologia in Mexico have now shown that a modified electrochemical deposition technique, combined with standard photolithography, can create integrated microthermoelectric coolers with high performance (Nat. Electron. https://doi. org/10.1038/s41928-018-0148-3; 2018).

The researchers built devices composed of four vertical layers: silicon substrate, bottom gold contact, thermoelectric elements and top-bridge gold contact. Bismuth tellurium compounds were used as the thermoelectrics and, unlike other related approaches, the thermoelectric elements were transferred to a gold electrolyte bath immediately after growth by electrochemical deposition. This created a protective gold layer on the elements, and allowed an interface with minimal resistance to be formed between the

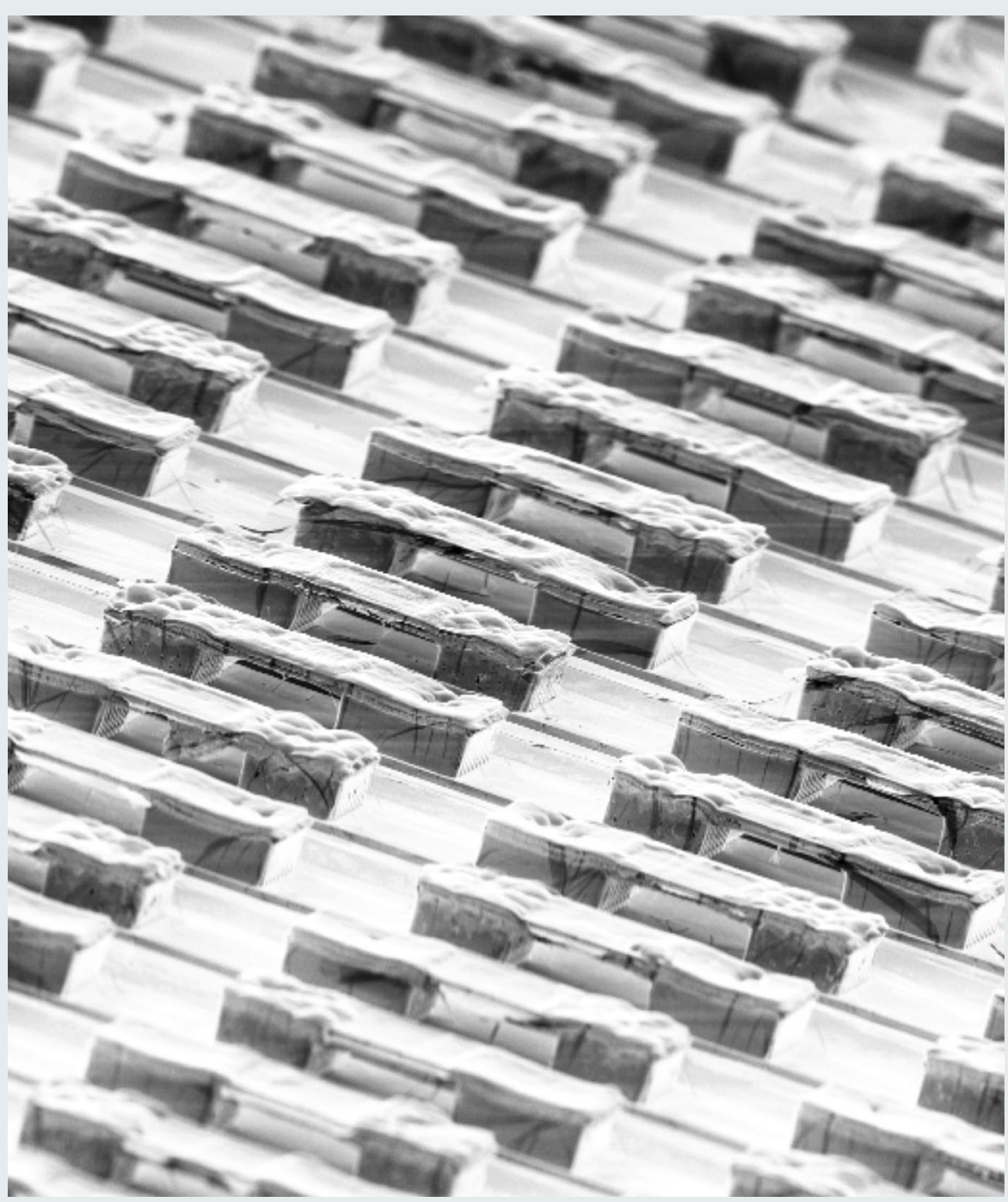

Credit: image courtesy of Guodong Li, IFW Dresden, Germany

thermoelectric legs and the top gold contact. With this approach, $\mathrm{Li}$ and colleagues were able to construct microthermoelectric devices that offer a fast cooling response time of $1 \mathrm{~ms}$, a high reliability of more than 10 million cycles and a cooling performance that is stable for more than 30 days.

The figure shows a scanning electron microscopy image of the integrated microthermoelectric device, which has a packing density of around 5,000 leg pairs per $\mathrm{cm}^{2}$. Each pair has an n-type semiconductor leg with a width of $30 \mu \mathrm{m}$ and a length of 40 $\mu \mathrm{m}$, and a p-type semiconductor leg with a width and length of $30 \mu \mathrm{m}$.

$\square$

Owain Vaughan

Published online: 12 October 2018 https://doi.org/10.1038/s41928-018-0154-5 